\title{
Correction to: Analysis and recommendations for building energy efficiency financing in Malaysia
}

\author{
Miroslav Lesjak • Kevin Hor • Mohd Khairil Rahmat
}

Published online: 19 June 2018

(C) Springer Nature B.V. 2018

\section{Correction to: Energy Efficiency (2018)}

https://doi.org/10.1007/s12053-017-9551-2

The original article has only two authors: Kevin Hor and Mohd Khairil Rahmat.

Miroslav Lesjak made contributions to the analysis presented in the paper. An oversight and confusion on the rights to the analysis work due to funding resulted to Miroslav Lesjak not added to the author group. The complete list of authors should be Miroslav Lesjak, Kevin Hor, and Khairil Rahmat.

The online version of the original article can be found at https://doi.org/10.1007/s12053-017-9551-2

M. Lesjak

IzvrstAn Consulting, Zagreb, Croatia

K. Hor $(\bowtie) \cdot$ M. K. Rahmat

UniKL British Malaysia Institute, Gombak, Selangor, Malaysia

e-mail: kev.hor@gmail.com 